ROSSETTO, C.A.V.; LIMA, T.M.; NAKAGAWA, J. Qualidade fisiológica e potencial de armazenamento de sementes de tomate submetidas ao condicionamento osmótico. Horticultura Brasileira, Brasília, v. 20, n. 4, p. 630-634, dezembro 2002.

\title{
Qualidade fisiológica e potencial de armazenamento de sementes de tomate submetidas ao condicionamento osmótico
}

\author{
Claudia Antonia Vieira Rossetto ${ }^{1}$; Tatiana de Moraes Lima ${ }^{1}$; João Nakagawa ${ }^{2}$ \\ ${ }^{1}$ UFRRJ, C. Postal 74511, 23.851-970 Seropédica - RJ, E-mail: cavrosse@ufrrj.br (Bolsista CNPq).; ${ }^{2}$ UNESP, C. Postal 237, 18.603-
}

970 Botucatu-SP (Bolsista CNPq).

\section{RESUMO}

Considerando a necessidade de verificar os efeitos de tratamentos de condicionamento osmótico na qualidade fisiológica e no potencial de armazenamento, foram avaliados três lotes comerciais de sementes de tomate representados pelos cultivares Quadrado Mix, Calmec VFN e Topmec. As sementes foram classificadas por peneiras de crivo circular de diâmetros de 3,5 e $4,7 \mathrm{~mm}$, passando cada lote a constituir um sublote e, em seguida, foram submetidas ao condicionamento osmótico. $\mathrm{O}$ delineamento experimental foi inteiramente casualizado em fatorial $2 \times 5 \times 6$ (condicionamento osmótico $\mathrm{x}$ sublote), com quatro repetições, por período de armazenamento. Por tratamento, as sementes foram imersas em soluções aeradas de polietileno glicol, a 0,0 e $-1,0 \mathrm{MPa}$, por sete dias, a $20^{\circ} \mathrm{C}$. As avaliações da qualidade fisiológica foram realizadas imediatamente após o condicionamento e aos 30; 60; 90 e 120 dias de armazenamento em potes plásticos e em condições de câmara fria. Os resultados permitiram concluir que imediatamente após o tratamento e até 60 dias de armazenamento, as sementes condicionadas apresentaram maior germinação e vigor, independente do sublote. Porém, após 120 dias de armazenamento, as sementes da cultivar Quadrado Mix, que foram condicionadas, apresentaram maior germinação e vigor, do que as sementes não condicionadas.

Palavras-chave: Lycopersicon esculentum, embebição, germinação.

\begin{abstract}
Evaluation of conditioning on the physiological quality and storability of tomato seeds

Tomato seeds of cv. Quadrado Mix, Calmec VFN and Topmec were classified by sieves of $3.5 \mathrm{~mm}$ and $4.7 \mathrm{~mm}$ diameter and submitted to osmotic conditioning. The experimental design was the completely randomized as a factorial 2x6 (osmotic conditioning $\mathrm{x}$ lot), for storage period, with four repetitions. The seeds were immersed in solutions supplied with air at $20^{\circ} \mathrm{C}$ in PEG 6000 solutions at 0.0 and $-1.0 \mathrm{MPa}$, during seven days. The results led to the conclusion that immediately after the treatment and up to 30 storage days, the conditioned seeds showed larger germination and vigor, independent of cultivar and diameter. However, after 120 storage days, the conditioned seeds of cv. Quadrado Mix showed larger germination and vigor than non-conditioned seeds.
\end{abstract}

Keywords: Lycopersicon esculentum, soaking, germination.

\section{(Recebido para publicação em 23 de outubro de 2001 e aceito em 20 de maio de 2002)}

$\mathrm{N}_{\mathrm{r}}^{\mathrm{a}}$ Ta cultura do tomate, o período compreendido entre a semeadura e a emergência das plântulas representa uma das fases críticas do ciclo da planta. Portanto, são desejáveis os tratamentos realizados antes da semeadura, visando a indução da semente, principalmente de híbridos (Mauromicale \& Cavallaro, 1995), a uma emergência rápida e uniforme (Frett et al., 1991; Pill et al., 1991) e a tolerância às condições adversas, tais como, deficiências hídricas nos substratos e baixas temperaturas, durante a germinação (Alvarado et al., 1987). Dentre estes, destaca-se o condicionamento osmótico, que consiste na pré-embebição das sementes em solução de potencial osmótico conhecido, de modo a controlar a hidratação nas sementes. O polietilenoglicol (PEG), soluto mais utilizado, abaixa o potencial hídrico das soluções osmóticas, contu- do pode limitar o oxigênio disponível para a germinação das sementes (Hardegree \& Emmerich, 1994).

O condicionamento de sementes de tomate pode ser feito empregando-se a metodologia do papel germitest umedecido com solução condicionante (Frett et al., 1991; Pill et al., 1991) ou a metodologia da solução condicionante aerada (Rossetto et al., 2001). Com a utilização da metodologia da solução condicionante aerada, Liptay \& Zariffa (1993) observaram redução no tempo de protrusão das sementes condicionadas em solução de PEG 6000, a -0,5 MPa. Além disso, Alvarado et al. (1987) verificaram que o condicionamento, empregando PEG 8000, a -1,25 MPa, por uma semana, a $20^{\circ} \mathrm{C}$, favoreceu o decréscimo do período de tempo de emergência no campo e não interferiu na porcentagem de germinação (protrusão da raiz primária) das sementes das cultivares estudadas. No entanto, Mauromicale \& Cavallaro (1995) constataram aumento da porcentagem de germinação (protrusão da raiz primária) das sementes condicionadas, sob potencial osmótico de -0,9 $\mathrm{MPa}$, com diferenças entre as cultivares; porém, não constataram efeito sobre o tempo médio de germinação. Também, Muhyaddin \& Wiebe (1989) verificaram maior emergência das plântulas obtidas de sementes condicionadas em solução aerada de PEG 4000, a -1,2 MPa.

Dos lotes de sementes de hortaliças, que têm sido condicionados, alguns são postos a germinam imediatamente e outros são submetidos a secagem e armazenados por meses antes da germinação. Dias et al. (1999), trabalhando com quiabo, observaram que após nove meses de armazenamento, houve au- 
Tabela 1. Dados médios, em porcentagem, de germinação, obtidos de seis sublotes de sementes de tomate dos cultivares Quadrado Mix, Calmec VFN e Topmec, de diâmetros de 3,5 e 4,7 mm, submetidos (C.C.) ou não (S.C.) ao condicionamento e avaliados aos 0; $30 ; 60$; 90 e 120 dias de armazenamento. Seropédica, UFRRJ. 2000.

\begin{tabular}{|c|c|c|c|c|c|c|c|}
\hline \multirow{2}{*}{$\begin{array}{c}\text { Sublotes } \\
\text { (Cultivar/Peneira) }\end{array}$} & \multirow{2}{*}{0} & \multirow{2}{*}{30} & \multirow{2}{*}{60} & \multicolumn{2}{|c|}{90} & \multicolumn{2}{|c|}{120} \\
\hline & & & & C.C. & S.C. & C.C. & S.C. \\
\hline Quadrado Mix /3,5 & $78,7 \mathrm{ab}$ & $74,2 \mathrm{a}$ & $82,4 a$ & $86,7 \mathrm{Aa}$ & $77,3 \mathrm{Bb}$ & $76,0 \mathrm{Aa}$ & 71,0 Bab \\
\hline Quadrado Mix /4,7 & $78,7 \mathrm{ab}$ & 82,7 a & 86,4 a & $83,3 \mathrm{Aa}$ & $78,0 \mathrm{Bb}$ & $76,0 \mathrm{Aa}$ & $69,5 \mathrm{Bb}$ \\
\hline Calmec VFN /3,5 & 75,5 b & 79,5 a & 77,0 a & $76,7 \mathrm{Aa}$ & $73,3 \mathrm{Ac}$ & $72,0 \mathrm{Ab}$ & $62,0 \mathrm{Ab}$ \\
\hline Calmec VFN /4,7 & $72,1 \mathrm{~b}$ & 77,7 a & 79,0 a & $73,3 \mathrm{Aa}$ & $78,7 \mathrm{Ab}$ & $62,6 \mathrm{Ab}$ & $57,0 \mathrm{Ab}$ \\
\hline Topmec /3,5 & 86,0 a & 85,4 a & $84,0 \mathrm{a}$ & $86,0 \mathrm{Aa}$ & $82,0 \mathrm{Aa}$ & $81,3 \mathrm{Aa}$ & $81,0 \mathrm{Aa}$ \\
\hline Topmec $/ 4,7$ & 83,0 a & 87,7 a & 80,0 a & $84,0 \mathrm{Aa}$ & $82,0 \mathrm{Aa}$ & $74,7 \mathrm{Aa}$ & 72,0 Aab \\
\hline Média C.C. & $80,9 \mathrm{~A}$ & $80,4 \mathrm{~A}$ & $83,4 \mathrm{~A}$ & & & & \\
\hline Média S.C. & 77,4 B & $81,9 \mathrm{~A}$ & $79,4 \mathrm{~A}$ & & & & \\
\hline
\end{tabular}

* Médias seguidas de mesma letra, maiúscula na linha e minúscula na coluna, não diferem entre si pelo Teste de Tukey a 5\% de probabilidade.

mento na porcentagem de plântulas normais obtidas das sementes do lote de qualidade inferior, tratada com PEG, a -0,8 MPa. Para Drew et al. (1997), avaliando lotes de sementes de cenoura e cebola, a porcentagem de protrusão de raiz primária de sementes submetidas ao pré-tratamento com PEG, não foi afetada pela secagem e pelo armazenamento, mas o desenvolvimento das plântulas foi prejudicado, sendo que o número de plântulas anormais aumentou durante $\mathrm{o}$ armazenamento. Em tomate, no entanto, Penãloza \& Eira (1993) verificaram que os efeitos dos tratamentos de hidratação e desidratação não persistem por longo período sob condições de armazenamento em sacos de papel e em ambiente sem controle.

Diante do exposto, o objetivo deste trabalho foi avaliar o efeito do condicionamento osmótico na qualidade fisiológica e no potencial de armazenamento de sementes de tomate.

\section{MATERIAL E MÉTODOS}

No trabalho, realizado de janeiro a outubro de 2000 , foram utilizados três lotes comerciais de sementes de tomate (Lycopersicon esculentum Mill.), representados pelas cultivares Quadrado Mix, Calmec VFN e Topmec, que permaneceram armazenadas em potes plásticos e em condições de $16^{\circ} \mathrm{C}$ de temperatura e $73 \%$ de umidade relativa do ar.

As sementes foram classificadas por peneiras de crivo circular de diâmetros de 3,5 mm (9/64") e 4,7 mm (12/64"), passando cada lote a constituir dois sublotes; em seguida, foram submetidas ao condicionamento osmótico, empregando soluções de polietileno glicol de peso molecular 6000 (PEG 6000), com os potenciais hídricos de 0,0 e -1,0 $\mathrm{MPa}$ (Villela et al., 1991). As avaliações foram feitas a 0; 30; 60; 90 e 120 dias de armazenamento.

O delineamento experimental foi inteiramente casualizado em fatorial $2 \times 5 \times 6$ (condicionamento osmótico $\mathrm{x}$ sublote), com quatro repetições, por período de armazenamento. Para isto, as amostras de aproximadamente $2,0 \mathrm{~g}$ de sementes, por sublote, foram devidamente identificadas e imersas em 200 $\mathrm{ml}$ de solução de $\mathrm{PEG}$, contidos em Erlenmeyer de $500 \mathrm{ml}$, vedados com chumaço de algodão, supridos com aeração constante, através de bomba de aquário e mantidos a $20^{\circ} \mathrm{C}$, por sete dias, com base na curva de absorção de água pelas sementes de tomate, obtida em ensaio preliminar. Após o período de embebição, as sementes foram colocadas para secar por 24 horas, sobre duas folhas de papel em condições de ambiente sem controle de temperatura e umidade relativa do ar.

As sementes de cada tratamento foram submetidas a testes para avaliação da qualidade, imediatamente após o condicionamento e aos 30; 60; 90 e 120 dias de armazenamento em potes plásticos e em condições de câmara fria. Na determinação do grau de umidade, quatro subamostras de aproximadamente 0,25 $\mathrm{g}$ de sementes foram submetidas ao método da estufa, a $105 \pm 3^{\circ} \mathrm{C}$, por 24 horas (Brasil, 1992). No teste de germinação, quatro subamostras de 50 sementes foram mantidas sob 8 horas de luz, à temperatura de $30^{\circ} \mathrm{C}$ e, sob 16 horas de escuro, à temperatura de $20^{\circ} \mathrm{C}$, sendo que as avaliações foram realizadas aos cinco e quatorze dias, seguindo-se a metodologia descrita em Brasil (1992). No teste de primeira contagem de germinação, foi considerada a porcentagem média de plântulas normais obtidas aos cinco dias do teste anterior.

A análise de variância foi realizada por lote e por tamanho das sementes. Os dados em porcentagem foram previamente transformados em $\operatorname{arcsen}\left(\right.$ x.100) ${ }^{1 /}$ ${ }^{2}$, mas os valores originais foram empregados nas Tabelas. A comparação das médias foi feita pelo teste Tukey, a 5\% de probabilidade.

\section{RESULTADOS E DISCUSSÃO}

As sementes de tomate que foram submetidas ao condicionamento osmótico apresentaram maior grau de umidade, apenas quando foram avaliadas imediatamente após o tratamento, independente do sublote. Porém, durante o período de armazenamento, não houve diferenças significativas entre os sublotes e os tratamentos (dados não apresentados).

$\mathrm{Na}$ avaliação realizada imediatamente após o condicionamento 
Tabela 2. Dados médios, em porcentagem, de total de plântulas anormais obtidos de seis sublotes de sementes de tomate das cultivares Quadrado Mix, Calmec VFN e Topmec, de diâmetros de 3,5 e 4,7 mm, submetidos (C.C.) ou não (S.C.) ao condicionamento e avaliados aos 0; 30; 60; 90 e 120 dias de armazenamento. Seropédica. UFRRJ. 2000.

\begin{tabular}{|c|c|c|c|c|c|c|}
\hline \multirow{2}{*}{$\begin{array}{c}\text { Sublote } \\
\text { (Cultivar/diâmetro) }\end{array}$} & \multicolumn{2}{|c|}{0} & \multirow{2}{*}{30} & \multirow[b]{2}{*}{60} & \multirow{2}{*}{90} & \multirow{2}{*}{120} \\
\hline & C.C. & S.C. & & & & \\
\hline Quadrado Mix /3,5 & $8,0 \mathrm{Ab}$ & $9,3 \mathrm{Aa}$ & $17,2 \mathrm{a}$ & $7,0 \mathrm{~b}$ & $6,0 a b$ & $13,3 a b$ \\
\hline Quadrado Mix /4,7 & $4,0 \mathrm{Bc}$ & $14,7 \mathrm{Aa}$ & $11,0 a b$ & $4,3 \mathrm{c}$ & $9,0 \mathrm{a}$ & $13,0 a b$ \\
\hline Calmec VFN /3,5 & $12,0 \mathrm{Aa}$ & $10,7 \mathrm{Aa}$ & $9,6 \mathrm{ab}$ & $10,0 \mathrm{a}$ & $8,7 \mathrm{a}$ & $13,9 a b$ \\
\hline Calmec VFN $/ 4,7$ & 10,4 Aa & $8,0 \mathrm{Aa}$ & $8,0 \mathrm{~b}$ & $7,3 \mathrm{~b}$ & $7,7 \mathrm{a}$ & $19,6 \mathrm{a}$ \\
\hline Topmec /3,5 & $2,0 \mathrm{Bc}$ & $10,7 \mathrm{Aa}$ & $10,3 a b$ & $11,2 \mathrm{a}$ & $4,7 \mathrm{~b}$ & $7,8 \mathrm{~b}$ \\
\hline Topmec 14,7 & $6,0 \mathrm{Bb}$ & $10,7 \mathrm{Aa}$ & $6,4 \mathrm{~b}$ & $11,3 \mathrm{a}$ & $5,0 \mathrm{~b}$ & $13,3 a b$ \\
\hline Média - C.C. & & & $12,3 \mathrm{~A}$ & $7,0 \mathrm{~A}$ & $5,0 \mathrm{~A}$ & $14,0 \mathrm{~A}$ \\
\hline Média - S.C. & & & $8,5 \mathrm{~B}$ & $9,9 \mathrm{~A}$ & $8,7 \mathrm{~A}$ & $12,9 \mathrm{~A}$ \\
\hline
\end{tabular}

* Médias seguidas de mesma letra, maiúscula na linha e minúscula na coluna, não diferem entre si pelo Teste de Tukey a 5\% de probabilidade.

osmótico, as sementes que foram condicionadas apresentaram maior porcentagem de germinação, independente do sublote (Tabela 1). Também, verificouse que independente do tratamento, as sementes da cultivar Topmec apresentaram as maiores porcentagens de germinação, embora estes valores não tenham diferido em relação aos obtidos pelas sementes da cultivar Quadrado Mix. Em sementes de tomate que foram condicionadas, Mauromicale \& Cavallaro (1995) constataram aumento da porcentagem de germinação (protrusão da raiz primária), com diferenças entre os lotes. Em relação às avaliações realizadas aos 30 e 60 dias de armazenamento, foi constatado que não houve diferenças significativas entre os sublotes e os tratamentos. Em quiabo, Dias et al. (1999) também observaram que não houve diferença entre os lotes e os procedimentos de condicionamento osmótico de sementes, até três meses de armazenamento.

Aos 90 e 120 dias de armazenamento, houve efeito da interação sublotes e tratamentos. As sementes da cultivar Quadrado Mix, que foram submetidas ao condicionamento, apresentaram maior porcentagem de germinação do que as que não foram condicionadas. Também, verificou-se que quando as sementes não foram submetidas ao condicionamento (testemunha), as da cultivar Quadrado Mix apresentaram menor porcentagem de germinação do que as sementes de Topmec, de diâmetro de 3,5 mm. Para Penaloza \& Eira (1993), os tratamentos de embebição controlada não foram eficientes em lotes de alto vigor, sendo que os efeitos não persistiram por longo período, sob condições de armazenamento em sacos de papel e em ambiente sem controle.

Em relação ao total de plântulas anormais, foi verificado que na avaliação realizada imediatamente após o condicionamento osmótico (Tabela 2), houve interação entre tratamentos e sublotes. As sementes da cultivar Quadrado Mix, de diâmetro de 4,7 mm e de Topmec, que foram submetidas ao tratamento de condicionamento, apresentaram menor porcentagem total de plântulas anormais. Aos 30 dias de armazenamento, as sementes condicionadas apresentaram maior porcentagem de plântulas anormais do que as não condicionadas, independente do sublote. Porém, a partir de 30 dias, não houve diferença entre sementes condicionadas e não condicionadas em relação à porcentagem de plântulas anormais, independente do sublote. Além disso, independente do tratamento, constatou-se que as sementes da cultivar Topmec apresentaram maior porcentagem de plântulas anormais, aos 60 dias de armazenamento, embora não tenha sido diferente das sementes da cultivar Calmec, de diâmetro de $3,5 \mathrm{~mm}$. Isto pode justificar o fato de não ter havido diferença significativa entre sublotes, quando foi observada a porcentagem de germinação, por ocasião desta avaliação (Tabela 1) e, aos 90 e 120 dias de armazenamento, estas sementes da cultivar Topmec apresentaram menor porcentagem de plântulas anormais, embora não tenha diferido das obtidas pelas sementes de outros sublotes.

Em relação ao desenvolvimento destas plântulas, foi observado na Tabela 3 que na avaliação realizada imediatamente após o condicionamento osmótico, as sementes da cultivar Quadrado Mix, de diâmetro de 4,7 mm e de Topmec, que foram submetidas ao tratamento de condicionamento, apresentaram menor porcentagem de plântulas deformadas, do que as que não foram condicionadas. Estes resultados concordam com os obtidos na Tabela 2, para o total de plântulas anormais, por ocasião da mesma avaliação. Em relação à avaliação realizada aos 30 dias de armazenamento, não houve diferenças significativas entre os sublotes e tratamentos, assim como também não houve para germinação (Tabela 1). Aos 60 e 120 dias de armazenamento, verificou-se que não houve diferença significativa entre os sublotes, independente de tratamentos. Para Drew et al. (1997), avaliando lotes de sementes de cenoura e cebola, verificaram que durante o armazenamento, a porcentagem de protrusão de raiz primária de sementes submetidas ao prétratamento com PEG, não foi afetada, porém, o desenvolvimento das plântulas foi prejudicado, em função do aumento do número de plântulas anormais. Para 
Tabela 3. Dados médios, em porcentagem, de plântulas anormais deformadas obtidos de seis sublotes de sementes de tomate dos cultivares Quadrado Mix, Calmec VFN e Topmec, de diâmetros de 3,5 e 4,7mm, submetidos (C.C.) ou não (S.C.) ao condicionamento e avaliado aos 0; 30; 60; 90 e 120 dias de armazenamento. Seropédica. UFRRJ. 2000.

\begin{tabular}{|c|c|c|c|c|c|c|c|}
\hline \multirow{2}{*}{$\begin{array}{c}\text { Sublotes } \\
\text { (Cultivar/diâmetro) }\end{array}$} & \multicolumn{2}{|c|}{0} & \multirow{2}{*}{30} & \multirow{2}{*}{60} & \multicolumn{2}{|c|}{90} & \multirow{2}{*}{120} \\
\hline & C.C. & S.C. & & & C.C. & S.C. & \\
\hline Quadrado Mix /3,5 & 6,0 Aa & 9,3 Aa & $4,0 \mathrm{a}$ & $5,7 \mathrm{a}$ & 3,3 Aa & $5,3 A b$ & $10,0 \mathrm{a}$ \\
\hline Quadrado Mix /4,7 & 3,3 Bab & $14,7 \mathrm{Aa}$ & $5,4 \mathrm{a}$ & $3,0 \mathrm{a}$ & 1,3 Bab & $10,0 \mathrm{Aa}$ & $8,8 \mathrm{a}$ \\
\hline Calmec VFN /3,5 & $8,0 \mathrm{Aa}$ & 9,3 Aa & $7,2 \mathrm{a}$ & $7,3 \mathrm{a}$ & 2,0 Bab & $8,0 \mathrm{Aa}$ & $11,1 \mathrm{a}$ \\
\hline Calmec VFN /4,7 & 7,3 Aa & 6,7 Aa & 9,3 a & $5,3 a$ & $0,0 \mathrm{Bb}$ & $4,7 \mathrm{Ab}$ & $11,8 \mathrm{a}$ \\
\hline Topmec /3,5 & $0,7 \mathrm{Bb}$ & $10,7 \mathrm{Aa}$ & 7,9 a & $5,3 a$ & $0,0 \mathrm{Bb}$ & $6,0 \mathrm{Aa}$ & $7,4 \mathrm{a}$ \\
\hline Topmec 14,7 & 4,7 Bab & $10,7 \mathrm{Aa}$ & $5,0 \mathrm{a}$ & $6,7 \mathrm{a}$ & $0,0 \mathrm{Bb}$ & $7,7 \mathrm{Aa}$ & 7,9 a \\
\hline Média - C.C. & & & $6,0 \mathrm{~A}$ & $3,6 \mathrm{~B}$ & & & $10,2 \mathrm{~A}$ \\
\hline Média - S.C. & & & $6,8 \mathrm{~A}$ & $7,6 \mathrm{~A}$ & & & $8,8 \mathrm{~B}$ \\
\hline
\end{tabular}

C.V.(\%) 21,9

${ }^{*}$ Médias seguidas de mesma letra, maiúscula na linha e minúscula na coluna, não diferem entre si pelo Teste de Tukey a 5\% de probabilidade.

Tabela 4. Dados médios, em porcentagem, de plântulas normais na primeira contagem obtidos de seis sublotes de sementes de tomate dos cultivares Quadrado Mix, Calmec VFN e Topmec, de diâmetros de 3,5 e 4,7 mm, submetidos (C.C.) ou não (S.C.) ao condicionamento e avaliados aos 0; 30; 60; 90 e 120 dias de armazenamento. Seropédica, UFRRJ. 2000.

\begin{tabular}{|c|c|c|c|c|c|c|c|}
\hline \multirow{2}{*}{$\begin{array}{c}\text { Sublotes } \\
\text { (Cultivar/diâmetro) }\end{array}$} & \multirow{2}{*}{0} & \multirow{2}{*}{30} & \multirow{2}{*}{60} & \multicolumn{2}{|c|}{90} & \multicolumn{2}{|c|}{120} \\
\hline & & & & C.C. & S.C. & C.C. & S.C. \\
\hline Quadrado Mix /3,5 & $37,4 \mathrm{ab}$ & $37,0 a b$ & $40,0 \mathrm{a}$ & 30,7 Aab & $25,3 \mathrm{Aa}$ & $30,6 \mathrm{Aa}$ & $18,0 \mathrm{Bbc}$ \\
\hline Quadrado Mix /4,7 & $37,7 \mathrm{ab}$ & $40,7 \mathrm{ab}$ & $40,7 \mathrm{a}$ & 26,0 Aab & $26,7 \mathrm{Aa}$ & 26,6 Aab & $13,5 \mathrm{Bc}$ \\
\hline Calmec VFN /3,5 & $34,0 \mathrm{bc}$ & $33,3 \mathrm{ab}$ & $32,7 \mathrm{a}$ & $15,3 \mathrm{Ab}$ & $16,0 \mathrm{Ab}$ & $19,3 \mathrm{Ab}$ & $19,0 \mathrm{Abc}$ \\
\hline Calmec VFN /4,7 & $29,4 \mathrm{c}$ & $31,2 \mathrm{~b}$ & $34,7 \mathrm{a}$ & $18,3 \mathrm{Ab}$ & $17,3 \mathrm{Ab}$ & $19,3 \mathrm{Ab}$ & $17,0 \mathrm{Abc}$ \\
\hline Topmec /3,5 & $44,4 \mathrm{a}$ & 43,4 a & $41,0 \mathrm{a}$ & $41,3 \mathrm{Aa}$ & $25,3 \mathrm{Ba}$ & $30,6 \mathrm{Aa}$ & $27,5 \mathrm{Aa}$ \\
\hline Topmec 14,7 & $40,4 \mathrm{a}$ & $40,7 \mathrm{ab}$ & $38,7 \mathrm{a}$ & $34,7 \mathrm{Aa}$ & $26,0 \mathrm{Aa}$ & $32,6 \mathrm{Aa}$ & $22,5 \mathrm{Bab}$ \\
\hline Média - C.C. & $41,4 \mathrm{~A}$ & $41,4 \mathrm{~A}$ & $41,9 \mathrm{~A}$ & & & & \\
\hline Media - S.C & $33,0 \mathrm{~B}$ & $34,0 \mathrm{~B}$ & $34,0 \mathrm{~B}$ & & & & \\
\hline C.V. (\%) & 9,3 & & & & & & \\
\hline
\end{tabular}

${ }^{*}$ Médias seguidas de mesma letra, maiúscula na linha e minúscula na coluna, não diferem entre si pelo Teste de Tukey a 5\% de probabilidade.

os autores, o potencial de armazenamento das sementes, que são submetidas a este tratamento é severamente limitado.

Em relação ao vigor, observou-se na Tabela 4, independente do sublote, que até 60 dias de armazenamento, as sementes que foram condicionadas apresentaram maior porcentagem de plântulas normais na primeira contagem, do que as que não foram tratadas. Aos 120 dias de armazenamento, as sementes dos sublotes Quadrado Mix, que foram submetidas ao condicionamento osmótico, apresentaram maior porcentagem de plântulas normais na primeira contagem, do que as que não foram condicionadas (Tabela 4), assim como maior germinação (Tabela 2).
De acordo com Nascimento (1998), a eficiência do condicionamento osmótico depende, dentre outros fatores, da qualidade inicial da semente, sendo que lotes de sementes com alta qualidade fisiológica não respondem ao "priming". Neste trabalho, as sementes das cultivares Quadrado Mix, Calmec e Topmec, apresentaram inicialmente, 31 ; 29 e 39\% de plântulas normais na primeira contagem e $75 ; 74$ e $83 \%$ de germinação, respectivamente.

Pelos resultados pode-se concluir que imediatamente após o tratamento e até 60 dias de armazenamento, as sementes condicionadas apresentaram maior germinação e vigor, do que as que não foram tratadas, independente do sublote. Porém, após 120 dias de armazenamento, as sementes da cultivar Quadrado Mix, que foram condicionadas, apresentaram maior germinação e vigor do que as não condicionadas. Esta diferença pode ser devido à qualidade fisiológica inicial ou à resposta dos genótipos a estes tratamentos.

\section{LITERATURA CITADA}

ALVARADO, A.D.; BRADFORD, K.J.; HEWITT, J.D.J. Osmotic priming of tomato seeds: effects on germination, field emergence, seedling growth, and fruit yield. Journal American Society Horticulture Science, v. 112, n. 3, p. 427-432, 1987.

BRASIL. Ministério de Agricultura e da Reforma Agrária. Regras para análise de sementes. Brasília: SNDA/ DNDV/ CLAV, 1992. 365 p. 
DIAS, D.C.F.S.; PAIXÃO, G.P.; SEDIYAMA, M.A.N.; CECON, P.R. Pré-condicionamento de sementes de quiabo: efeitos na qualidade fisiológica e no potencial de armazenamento. Revista Brasileira de Sementes, v. 21, n. 2, p. 224-231, 1999.

DREW, R.L.K.; HANDS, L.J.; GRAY, D. Relating the effects of priming to germination of unprimed seeds. Seed Science and Technology, v. 25, n. 3, p 537-548, 1997.

FRETT, J.J.; PILL, W.G.; MORNEAU, D.C. A comparison of priming agents for tomato and asparagus seeds. HortScience, v. 26, n. 9, p. 1158 1159, 1991.

HARDEGREE, S.P.; EMMERICH, W.E. Seed germination response to polyethylene glycol solution depth. Seed Science and Technology, v. 22, n. 1, p. 1-7, 1994.
LIPTAY, A.; ZARIFFA, N. Testing the morphological aspects of polyethylene glycolprimed tomato seeds with proportional odds analysis. HortScience, v. 28, n. 9, p. 881-883, 1993.

MAUROMICALE, G.; CAVALLARO, V. Effects of seed osmopriming on germination of tomato at different water potential. Seed Science and Technology, v. 23, n. 2, p. 393-403, 1995.

MUHYADDIN, T.; WIEBE, H.J. Effects of seed treatments with polyethylene glycol on emergence of vegetable crops. Seed Science and Technology, v. 17, n. 1, p. 49-56. 1989.

PENÃZOLA, A.P.S.; EIRA, M.T.S. Hydration dehydration treatments on tomato seeds (Lycopersicon esculentum Mill). Seed Science and Technology, Zurich, v. 21, n. 3, p. 309-316, 1993.
PILL, W.G.; FRETT, J.J.; MORNEAU, D.C. Germination and seedling emergence of primed tomato and asparagus seeds under adverse conditions. HortScience, v. 26, n. 9, p. 1160-1162, 1991.

ROSSETTO, C.A.V.; LIMA, T.M.; NAKAGAWA, J. Avaliação da aplicação de fungicida e do condicionamento osmótico durante o armazenamento de sementes de tomate. Revista Brasileira de Sementes, v. 23, n. 2, 2001 (no prelo).

VILLELA, F.A; FILHO, L.D.; SEQUEIRA, E.L. Tabela de potencial osmótico em função da concentração de polietileno glicol 6000 e da temperatura. Pesquisa Agropecuária Brasileira, Brasília, v. 26, n. 11/12, p. 1957-1968, 1991. 